\title{
Table of authorities
}

\section{CASES}

Angas Law Services Pty Ltd (in liq) v Carabelas [2005] HCA 23; (2005) 226 CLR 507 250, 266

Australian Securities and Investments Commission (ASIC) v Macdonald (No 11) [2009] NSWSC 287; (2009) 256 ALR 199 221, 226

Barings plc and Others, Re (No 5) [1999] 1 BCLC 433 262, 267

Bath v Standard Land Co Ltd [1911] 1 Ch 618 212, 225

BCE Inc v 1976 Debentureholders 2008 SCC $69 \mathbf{2 2 4}$

Credit Lyonnais Bank Nederland v Pathe Communications Corp CA No 12150 , 1991 Del. Ch. LEXIS 215 (Del Ch 1991) 212, 215

Gippsland Coastal Board v South Gippsland Shire Council [2008] VCAT 1545 311, 336

Gray v Minister for Planning [2006] NSWLEC $720 \quad \mathbf{3 1 1 ,} 336$

Hutton v West Cork Railway Co (1883) 23 Ch D 654 210, 225

J E Hurdley \& Son Ltd (in liq), In re [1941] NZLR $686 \quad$ 212, 225

Kinsela v Russell Kinsela Pty Ltd (in liq) (1986) 4 ACLC 215; (1986) 4 NSWLR 722 212, 225

Linney (H) \& Co Ltd, Re [1925] NZLR 907 212, 225

Louis K Liggett Company v Lee 288 US 517 (1933) 5, 31

Minister for the Environment and Heritage v Queensland Conservation Council Inc [2004] FCAFC 190; (2004) 139 FCR $24 \quad 311,336$
New World Alliance Pty Ltd, Re; Sycotex Pty Ltd v Baseler [No 2] (1994) 122 ALR 531; (1994) 51 FCR 425 212, 225 Nicholson v Permakraft (NZ) Ltd (in liq) [1985] 1 NZLR $242 \quad 212$

Paramount Communications Incorporated v Time Incorporated 571 A 2d 1140 (Del. 1989), quoting Unocal Corporation v Mesa Petroleum Co 493 A 2d 946, 955 (Del.1989) 207, 225

Parke v Daily News Ltd [1962] 2 All ER 929; [1962] 2 Ch 927 210, 225

Peoples Department Stores Inc (Trustee of) v Wise [2004] 3 SCR 461; 2004 SCC $68182,194,205,206,210,219,224$, 225, 226

Pepper v Litton 308 US 295 (1939) 211, 225 Queensland Conservation Council Inc v Xstrata Coal Queensland Pty Ltd [2007] QCA 338 311, 336

R v Broadcasting Standards Commission; Ex parte BBC [2001] QB 885; [2000] EWCA 59 311, 336

Revlon Inc v MacAndrews \& Forbes Holdings Inc 506 A 2d 173 (1986) 205, 224

Salomon v Salomon \& Co Ltd [1897] AC 22 105, 126

Sosa v Alvarez-Machain 542 US 692 (2004) 309, 336

Spies v R [2000] HCA 43; (2000) 201 CLR 603 212, 225

Teck Corp v Millar (1972) 33 DLR (3d) 288, endorsed in Peoples Department Stores Inc (Trustee of) v Wise [2004] 3 SCR 461; 2004 SCC $68 \quad \mathbf{2 0 6}, 225$

The Bell Group Ltd (in liq) v Westpac Banking Corp (No 9) [2008] WASC 239 212, 213, 225 
Walker v Wimborne (1976) 137 CLR 1212,225

White v Director of Military Prosecutions [2007] HCA 29; (2007) 231 CLR 570161,167

Wildlife Preservation Society of Queensland Proserpine/Whitsunday Branch Inc v Minister for the Environment \& Heritage [2006] FCA 736; (2006) 232 ALR 510 311, 336

Wincham Shipbuilding, Boiler \& Salt Company, In re (Poole, Jackson \& Whytes' Case) (1878) 9 Ch D 322 212, 225

YL v Birmingham City Council [2007] UKHL 27; [2007] All ER (D) 207 (Jun) 311, 336

\section{LEGISLATION}

\section{Australia}

Charter of Human Rights and Responsibilities Act 2006 (Vic) s 4(1) 311, 336 ss 38-39 312, 336

Corporate Code of Conduct Bill 2000 (Cth) $\mathbf{2 2 5}$

Corporate Code of Conduct Bill 2004 (Cth) 213, 215-216, 225-226

ss 7-13 215, 226

s 15(6) 216, 226

s 17(1) 215, 226

Part 3 216, 226

Part 4 216, 226

Corporations Act 2001 (Cth) 142-143, 180, 194-5, 218, 225-226, 338

s $128 \quad \mathbf{2 2 6}$
s $129 \quad \mathbf{2 2 6}$
s $180 \quad \mathbf{2 2 0 ,} \mathbf{2 2 6}$
s $180(2) \quad \mathbf{2 1 9 ,} \mathbf{2 2 6}$
s 299(1)(f) $\quad \mathbf{1 8 9 , \mathbf { 1 9 5 }}$
s 299A $\quad \mathbf{1 8 9 , \mathbf { 1 9 5 }}$
S 299A(1) $\quad \mathbf{1 8 9 , \mathbf { 1 9 5 }}$
s 299A(3) $\quad \mathbf{1 9 5}$

Part 5.8A (Employee Entitlements) 211, 255

s 1013DA 184, 195

s 1013D(1)(i) 184, 195, 333, 338

s 1324 180, 194

Human Rights Act 2004 (ACT)

s 40D 162, 167, 312, 336

\section{Canada}

Canada Business Corporations Act (1985) 205, 210 s 122(1)(a) 205, 225

s 122(1)(b) 206, 225

\section{United Kingdom}

Combined Code on Corporate Governance (UK) 56, 178, 179, 194 s 2, E. $1 \quad$ 179, 194

Companies Act 1985 (UK)

$$
\begin{array}{ll}
\text { s } 309 & \mathbf{2 5 5}-\mathbf{2 5 7} \\
\text { s 309(1) } & \mathbf{2 1 0 ,} \mathbf{2 2 5}, \mathbf{2 5 6} \\
\text { s 309(2) } & \mathbf{2 1 0 ,} \mathbf{2 2 5} \\
\text { s } 719(1) & \mathbf{2 1 0 ,} \mathbf{2 2 5}
\end{array}
$$

Companies Act 2006 (UK) xiii, 142, 135, 165, 181-182, 188, 194-195, 199, 210, 212, 214, 216, 219, 225-226, 229-232, 235, 238-240, 242-246, 248, 250-252, 256-258, 261-263, 265-267, 301,336

s $170 \quad 247$

s 170(1) 240, 265, s 172 182, 194, 232, 249-251, 253, 255-258, 265-266, 314, 336 s 172(1) 219, 226, 237, 253, 255, 261, 265-267
s 172(1)(d) 219, 226
s 172(2) 244, 249, 266
s 172(3) 212, 213, 225
s $174 \quad 250$
s $239 \mathbf{2 6 1 , 2 6 7}$
s 239(1) 250, 266
s 239(2) 250, 266
s 239(3) 250, 266
s 239(4) 250, 266 


\begin{tabular}{|c|c|}
\hline s 247(1) & 257,267 \\
\hline s $247(2)$ & 257,267 \\
\hline s 247(4) & 257,267 \\
\hline s $260 \quad 26$ & 61,267 \\
\hline s 261 & 61,267 \\
\hline s $262 \quad 26$ & 61,267 \\
\hline s 263 & 61,267 \\
\hline s $264 \quad 26$ & 61,267 \\
\hline s $417 \quad 25$ & $51,258-259,267$ \\
\hline s 417(1) & 260,267 \\
\hline s $417(2)$ & $233,251,261-262,265-267$ \\
\hline s 417(5) & $188,195,219,226,314$ \\
\hline 336 & \\
\hline s $417(5)(\mathrm{c}$ & c) 294,301 \\
\hline s 417(6) & 188,195 \\
\hline s 417(7) & 260,267 \\
\hline s 46326 & 61,267 \\
\hline Part 14 & 135,165 \\
\hline
\end{tabular}

(UK) 232, 236, 244

Corporate Responsibility (CORE) Bill

(UK) 213-215, 225

cl 2 214-215, 225

Corporate Responsibility Bill 2003

(UK) 225

Human Rights Act 1998 (UK) 311-312, 336

$$
\begin{array}{ll}
\text { s } 6(3) & \mathbf{3 1 1 ,}, \mathbf{3 3 6} \\
\text { ss } 6-8 & \mathbf{3 1 2 ,}, 336
\end{array}
$$

Occupational Pension Schemes

(Investment) Regulations 1996 (UK)

reg 11A 184, 195

Pensions Act 1995 (UK)

s 35 184, 195

\section{United States of America}

Alien Tort Claims Act 1789 309, 355

Corporate Code of Conduct Bill $2000 \mathbf{2 2 5}$

National Environmental Policy Act 1969336

Sarbanes-Oxley Act 2002191

Securities and Exchange Commission

Regulation S-K

Item $101 \quad$ 190, 195, 196
Item $103 \mathbf{1 9 0 , 1 9 6}$

Item $303 \mathbf{1 9 0 , 1 9 6}$

US Sentencing Guidelines for

Organizational Defendants $\mathbf{1 6 2}$

\section{TREATIES, CONVENTIONS AND OTHER \\ INTERNATIONAL \\ INSTRUMENTS}

EU Accounts Modernisation

Directive $\mathbf{1 8 7}$

EU Directive 2003/51/EC 187-188, 195

EU Directive 2006/46/EC 188, 195

European Convention on Human

Rights 306

International Convention on the

Elimination of all Forms of Racial

Discrimination 169

International Covenant on Civil and Political Rights 306

International Labor Organization

Tripartite Declaration of Principles

Concerning Multinational

Enterprises 9, 31, 55, 326, 340

OECD Guidelines for Multinational

Enterprises 15, 31, 40, 55, 144, 177, 186-187, 278, 326, 340

OECD Principles of Corporate

Governance 26, 31, 71, 177, 187, 278

Treaty of Westphalia $1648 \mathbf{3 4 8}$

TRIPS: WTO Agreement on Trade-

Related Aspects of Intellectual Property

Rights, $1994 \quad 307$

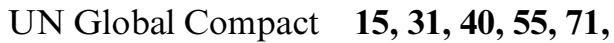
155, 192, 288, 292, 316, 318, 326, 327, 328, 340, 341

UN Norms on the Responsibilities of Transnational Corporations and Other Business Enterprises with Regard to Human Rights (UN Norms) 30,302 , 304, 308, 317-324, 327, 329, 334, 343, 355, 372 
Art $1 \quad 317,336$

Art 15 318, 336

Art 16 318, 336

Art 17 318, 336
Art 18 318, 336

Art 19 318, 336

Universal Declaration of Human

Rights 304-305 